Forum Articles

\title{
The domestication hypothesis for dogs' skills with human communication: a response to Udell et al. (2008) and Wynne et al. (2008)
}

\author{
Brian Hare $^{\mathrm{a}, *}$, Alexandra Rosati ${ }^{\mathrm{a}}$, Juliane Kaminski ${ }^{\mathrm{b}}$, Juliane Bräuer ${ }^{\mathrm{b}}$, Josep Call ${ }^{\mathrm{b}}$, Michael Tomasello ${ }^{\mathrm{b}}$ \\ ${ }^{a}$ Department of Evolutionary Anthropology \& Center for Cognitive Neuroscience, Duke University, Durham, NC, U.S.A. \\ ${ }^{\mathrm{b}}$ Max Planck Institute for Evolutionary Anthropology, Leipzig, Germany
}

\section{A R T I C L E I N F O}

Article history:

Received 7 February 2009

Initial acceptance 15 June 2009

Final acceptance 25 June 2009

Available online 29 August 2009

MS. number: AF-09-00115R

Keywords:

cognitive evolution

dog cognition

domestication

social cognition

Domestic dogs have special skills in comprehending human communicative behaviours (Hare \& Tomasello 2005; Miklosi 2008). Dogs across a range of breeds use human communicative cues such as pointing or physical markers to find food that is hidden in one of two hiding places (controls rule out the use of olfactory cues; Cooper et al. 2003; Hare \& Tomasello 2005; Miklosi \& Soproni 2006). In direct comparisons, dogs are even more skilled than chimpanzees at using human communicative cues when searching for food (Hare et al. 2002; Bräuer et al. 2006). Moreover, a number of studies suggest that dogs understand human gestures communicatively, as a number of possible low-level explanations have been ruled out (e.g. only responding to movement; reflexively coorienting; using only familiar cues, etc.; Hare et al. 1998; Agnetta et al. 2000; Soproni et al. 2001; Miklosi 2008).

The sophistication that dogs show in using human communicative cues led researchers to investigate the origins of these abilities and to conclude that these social skills are not simply inherited from wolves nor are they simply learned as a result of exposure to humans in ontogeny, but rather they have evolved as a result of domestication (Hare \& Tomasello 2005). Although wolves can potentially learn to use human communicative cues (much like nonhuman primates), they do not show these skills as young

\footnotetext{
* Correspondence: B. Hare, Department of Evolutionary Anthropology \& Center for Cognitive Neuroscience, Duke University, Durham, NC 27708, U.S.A.

E-mail address: b.hare@duke.edu (B. Hare).
}

puppies and must be explicitly trained to express dog-like skills in response to human communicative cues (Agnetta et al. 2000; Hare et al. 2002; Viranyi et al. 2008). This conclusion holds even for wolves reared in identical conditions with a group of dogs for the purpose of comparing their social skills with humans. In summary, it is unlikely that dogs simply inherited their unusual skills from their last common ancestor with wolves (Viranyi et al. 2008).

Additionally, dogs develop their ability to use human communicative cues, such as pointing cues or gaze cues, as young puppies regardless of rearing history. Even puppies as young as 6-9 weeks of age can use a human's communicative cues, including unadopted puppies still living with their littermates and having little exposure to humans beyond routine care (Hare et al. 2002; Riedel et al. 2008; Viranyi et al. 2008). This suggests that dogs' use of human communicative cues does not require extensive exposure to humans (e.g. Hare et al. 2002).

These two major findings led researchers to explore the possibility that dogs evolved their unusual ability to use human communicative cues not only during, but as a direct result of, domestication (Miklosi et al. 2003; Hare \& Tomasello 2005). Comparisons between experimentally domesticated foxes and a matched control line of foxes support the hypothesis that domestication, or selection against aggression towards humans, can, as a by-product, lead to enhanced skills in comprehending human gestures. Experimental foxes bred over 40 generations to approach humans without fear were more skilled at using human gestures than were control foxes that were not bred over the same 
period for their reaction to humans. Crucially, the control and experimental foxes participated in the experiments at similar levels (i.e. made equal numbers of choices within test sessions) and, when tested with a nonsocial cue, the control foxes were actually more skilled than the experimental foxes, effectively ruling out the possibility that the control foxes were too fearful or unmotivated to participate in all human-led tasks (Hare et al. 2005). The findings from these experiments with foxes provide further support that the domestication of dogs actually enhanced their ability to use communicative cues provided by humans.

Recently, two papers challenge the ideas (1) that dogs outperform wolves in using human communicative gestures (Udell et al. 2008) and (2) that dogs require very limited human exposure to show initial skill in using such communicative cues (Wynne et al. 2008). In the first of these studies, Udell et al. (2008) present findings suggesting that wolves are more skilled than dogs in using human communicative cues in a food retrieval task. Specifically they found that wolves were more skilled than shelter dogs and pet dogs tested outdoors, and equally as skilled as pet dogs tested indoors. In a second study, Wynne et al. (2008) critiqued an experiment by Riedel et al. (2008) that examined the use of human communicative cues by dog puppies. Riedel and colleagues reported a series of three studies showing that domestic dog puppies comprehended a human pointing gesture as early as 6 weeks of age. Wynne and colleagues suggest that this study did not rule out the possibility that human experience plays a decisive role. In both papers this team of authors claims that their evidence and reanalysis refute the domestication hypothesis, and they suggest that differences among canid species in social skills is largely due to environmental factors during rearing rather than being a result of dog domestication.

To evaluate the evidence presented in these studies, we first discuss several methodological concerns that we have about the approach of Udell et al. (2008), then we reanalyse their data based on these methodological concerns. We also present a test of shelter dogs naïve to cognitive testing to examine whether it is the case that shelter dogs are less skilled at using human communicative cues than other groups of dogs. Finally, we directly rebut the critique of Wynne et al. (2008) and argue that there remains no evidence of significant differences in performance between dogs of different ages in their use of human communicative cues. We conclude that the domestication hypothesis remains the best explanation for dogs' special skills for communicating with humans.

\section{REANALYSIS OF UDELL ET AL. (2008): ARE WOLVES MORE SKILLED THAN DOGS?}

In this section we describe the method of Udell et al. (2008), highlight important methodological differences with previous studies and re-examine their data. The authors kindly provided us with a trial-by-trial data set of both studies so that we could use a more conventional data analysis to re-examine the performance of their subjects.

Udell et al. (2008) examined the use of a pointing cue by five experimental groups of canids (one group of wolves, four groups of dogs), each with eight subjects of adult age. Unlike Hare et al. (2005) and Viranyi et al. (2008), none of the subjects were reared for the purposes of the experiment, so the authors could not be sure of the subjects' previous relevant experiences. This raises the question of how much previous experience the wolves had with performing human-directed tasks; this is particularly important given that these wolves had been used in public education shows and had been intensively exposed to humans. The wolves in Udell et al.'s (2008) study, like those in Hare et al. (2002) and Viranyi et al.
(2008), were tested by caregivers in familiar outdoor enclosures, but unlike in Hare et al.'s (2002) study, the caregiver in Udell et al.'s (2008) study tested the wolves while standing inside the subjects' enclosure. Three of the dog groups consisted of family-reared pet dogs that were tested by different combinations of familiar or unfamiliar experimenters and testing locations: dogs in the "home unfamiliar' group were tested at their homes by an experimenter; dogs in the 'outdoor familiar' group were tested in a less familiar outdoor location by a familiar human; and dogs in the 'outdoor unfamiliar' group were tested in a less familiar outdoor location by an experimenter. The final group consisted of dogs living at a local shelter that were tested in a less familiar room by an unfamiliar human experimenter.

Unlike all previous studies, Udell et al. (2008) gave subjects four warm-up trials in which they placed food on top of one of two hiding locations in view of subjects so that, once placed, the food was visible to the subject (usually in a warm-up phase with primates or dogs, food is hidden under different hiding locations as the subjects watch). During this warm-up, food was always completely visible and all subjects correctly retrieved the visible food in all four trials. After the warm-up, all subjects were then given 10 experimental test trials. However, the methodology used in the test trials was very different from that of previous studies. In Udell et al.'s (2008) study, no food was hidden, but rather the experimenter pointed and "when a subject indicated a correct choice, the experimenter clicked [a clicker device] and then dropped a piece of food on the chosen container' (page 3 ). This task differs greatly from the traditional method in which a human indicates the location of hidden food to a dog (reviewed in: Hare \& Tomasello 2005; Miklosi 2008).

The performance of each individual subject was compared to chance using binomial probabilities (authors assigned chance to $50 \%$, one-sample $t$ tests, one-way ANOVAs and post hoc comparisons (Bonferroni tests). In addition to the experimental trials, after every second experimental trial, a control trial was run that was identical to the experimental trial with the exception that no pointing cue was provided (these trials acted as control for olfactory cues that the subjects might be using). Unlike other studies, if subjects made incorrect choices for more than three trials consecutively they were given another warm-up trial in which food was placed on one of the two hiding locations as the animal watched; this was done to rule out the possibility that the subjects' failures were due to lack of food motivation.

Based on their analysis of the data, Udell et al. (2008) concluded that the performance of the five groups of subjects differed significantly from one another. Wolves and dogs tested indoors were the only groups to use the pointing gesture at above-chance levels, and wolves even significantly outperformed the shelter dogs in their use of the human pointing gesture. A follow-up study (Udell et al. 2008) compared two groups of dogs for their ability to follow a human point; one group was tested while they were behind a fence while the other group was tested while both the experimenter and subject were on the same side of the fence. Udell et al. (2008) reported that the dogs tested behind fences made more incorrect choices than did the group with no fence.

In describing the methods of Udell et al. (2008) we have highlighted several methodological differences between their work and previous studies (e.g. reviewed in Miklosi 2008) regarding test procedures. However, the most important difference concerns the coding of trials and subsequent data analysis, which we argue are statistically invalid. Although not stated explicitly in the paper, we now know that subjects were coded in both studies as making an incorrect choice not only if they chose the cup that the experimenter did not point at, but also in trials in which they made no choice at all (C. Wynne, personal communication). This is 
a particularly problematic method (and different from all previous studies; reviewed in Miklosi 2008) given that their statistical analysis relies on using $50 \%$ as random choice. In this system of scoring, 'incorrect' choices have a greater expected probability than correct choices because they include both the choice of the incorrect cup and no choice (no participation). To use an analogy, this scoring procedure would be like flipping a coin and considering heads as correct, tails as incorrect, and trials without flipping as incorrect also (e.g. it is a truism that there is $0 \%$ chance that a coin will land heads if it is never flipped). In previous studies the exclusion or repetition of no-choice trials has been the standard, because in no-choice trials, subjects often become distracted or even leave the testing area (i.e. temporarily losing motivation to solve the problem).

We now present the results of the same data using an almost identical analysis to that originally presented in Udell et al. (2008) but following the more conventional method of examining separately (1) participation (making a choice or not) and (2) the level of correct choices (choosing the cup indicated by a point instead of the one ignored). Figure 1 presents the mean percentage of trials from Udell et al.'s (2008) first experiment in which subjects from each of the five groups actually participated by approaching and making a choice in the 10 experimental trials. Our one-way ANOVA revealed a significant difference in the level of participation between the five groups $\left(F_{4,35}=9.06, P<0.001\right)$. Post hoc comparisons (Bonferroni tests) revealed that the dog home unfamiliar group participated significantly more often than the other three dog groups, but did not differ from the wolf group $(P<0.05$ for all significant tests). In addition, the wolf group participated significantly more often than the dog outdoor familiar and shelter dog groups $(P<0.05)$. Wolves also participated marginally more often than the dog outdoor unfamiliar group $(P=0.054)$.

Figure 2 presents the mean percentage of trials in which subjects that participated chose the container indicated by the experimenter over the incorrect container (the one that was not indicated). A one-way ANOVA revealed no significant differences between the five groups' performance in choosing the correct container in trials in which they participated $\left(F_{4,35}=0.308\right.$,

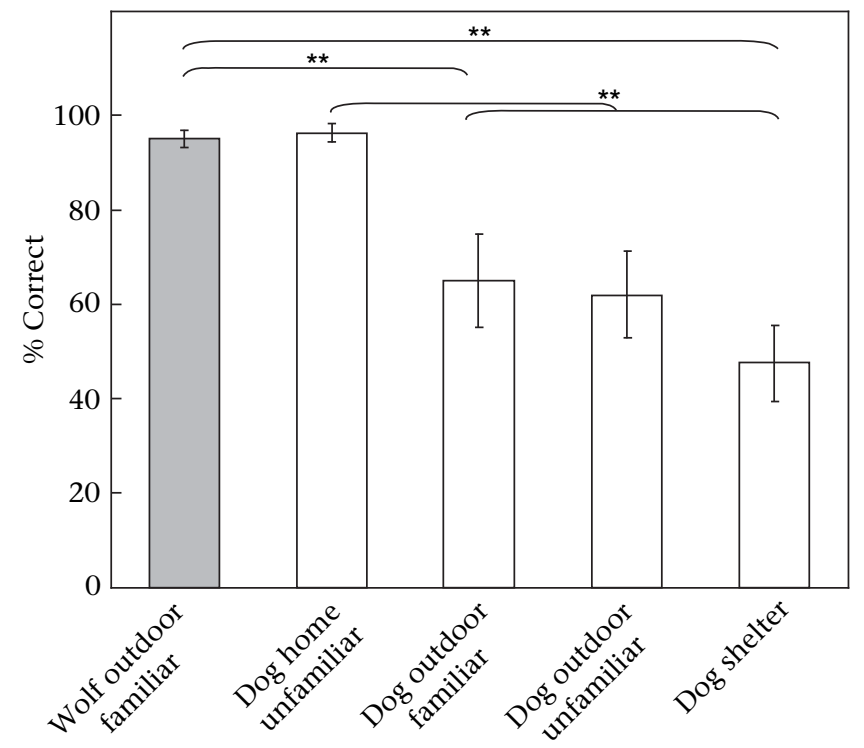

Figure 1. Mean \pm SE percentage of trials (out of 10 trials) in which subjects from the five experimental groups in Udell et al. (2008) made a choice by touching one of two choice options within 3 min. ${ }^{* *} P<0.05$ (post hoc comparisons between all groups, Bonferroni correction).

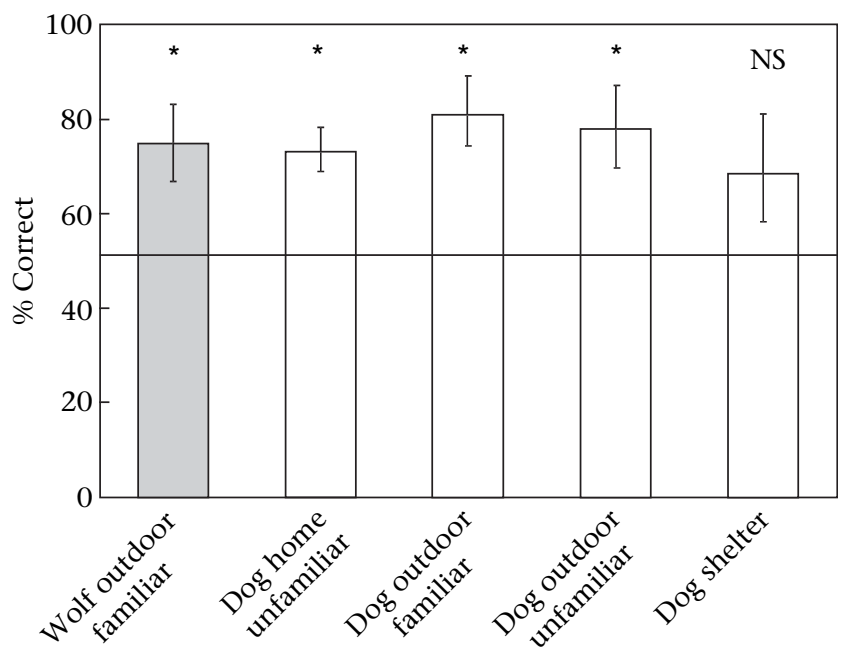

Figure 2. Mean \pm SE percentage of correct choices for one of two hiding locations by subjects in the five experimental groups in Udell et al. (2008). The line represents the level of chance performance. ${ }^{*} P<0.05$ (one-sample $t$ tests comparing each group to chance or $50 \%$ ).

$P=0.871)$. One-sample $t$ tests revealed that all groups but the shelter dog group chose the container indicated by a point at above-chance levels in trials when they made a choice (wolf: $t_{7}=2.992, P<0.02$; dog home unfamiliar: $t_{7}=4.32, P<0.003 ;$ dog outside familiar: $t_{7}=4.344, P<0.03$; $\operatorname{dog}$ outside unfamiliar: $t_{7}=3.422, P<0.011$; shelter dog: $\left.t_{7}=1.46, P<0.19\right)$. Figure 3 presents the mean percentage of trials from Udell et al.'s (2008) second experiment, in which the seven dogs from both groups $(N=14)$ participated in and made correct choices. As in the first experiment, while the two groups differed in the number of trials in which they participated $\left(t_{12}=2.43, P<0.05\right)$, their actual performance was identical in trials where a choice was made $\left(t_{11}=0.11\right.$, $P=0.92$ ). One dog was excluded from our new analysis because it never made a single choice; surprisingly, it was still counted as a subject in the original publication.

In direct opposition to the conclusions of Udell et al. (2008), wolves did not outperform dogs in using human communicative

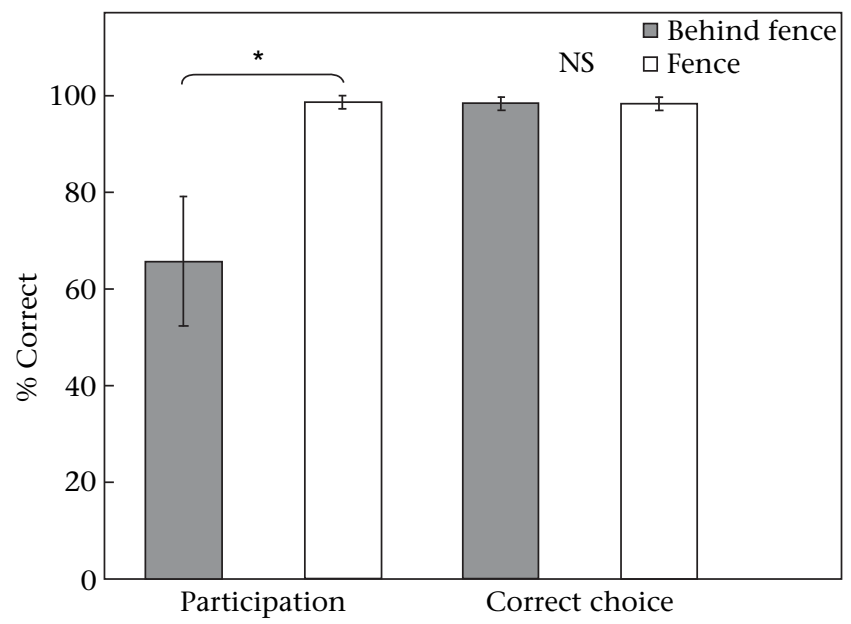

Figure 3. Mean $\pm \mathrm{SE}$ percentage of trials (out of 10 trials) in which dogs that were tested behind a fence and dogs that were not tested behind a fence participated in choice tests (by choosing one of two locations) and chose the correct location in Udell et al.'s (2008) study. ${ }^{*} P<0.05$ (independent $t$ tests). 
cues; the wolves were only more willing to participate in trials. Thus, there is no evidence from the data of Udell et al. (2008) that adult wolves outperform adult dogs using a human pointing gesture (making the results highly similar to those reported for mature wolves in Viranyi et al. 2008). Closer examination of the methods used to test each group probably explains the observed differences in participation of the subjects across groups. Both the wolf group and dog home unfamiliar group were tested in their daily surroundings in which they normally interacted, while the other three dog groups, which had significantly lower participation, were tested in more unfamiliar or even novel areas. This raises the distinct possibility that the dogs tested in more unfamiliar locations were often more interested in exploring their new surroundings than in attending to the task. In contrast, in both Hare et al. (2002) and Viranyi et al. (2008), the testing contexts for wolves and dogs were highly similar since subjects of both species were highly familiar with their testing location. Finally, testing a dog with a fence between the experimenter and dog made absolutely no difference in the use of the human pointing cue; again, it only affected the dogs' willingness to participate in the task.

The one curious result in our reanalysis of Udell et al.'s data (2008) was our finding that shelter dogs did not perform above chance as a group, even after we separated the two measures of choice and participation. This raises the possibility that shelter dogs, for temperamental or rearing history reasons, were less skilled in using human communicative cues than were subjects in the other groups (although the shelter dog group did not differ significantly from the other groups that used pointing at abovechance levels).

\section{EXPERIMENT: CAN SHELTER DOGS USE HUMAN COMMUNICATIVE CUES?}

Even after our reanalysis of Udell et al. (2008), it is possible that shelter dogs as a group may be different from other groups of dogs since they did not use a human pointing cue at above-chance levels. However, it is also possible that this negative finding is a result of the small sample of shelter dogs tested $(N=8)$, the small number of trials in which subjects actually participated (mean participation $<50 \%$ of trials), and the unconventional testing method used. Therefore, we tested a larger sample of shelter dogs to determine how skilful they are at using human communicative cues. Importantly, we tested them with the conventional object-choice methods used in virtually all other similar studies (e.g. reviewed in: Hare \& Tomasello 2005;) rather than with the methods of Udell et al. (2008).

\section{Methods}

Twenty-three domestic dogs residing in a no-kill dog shelter in Germany were tested. Twelve of the dogs were suspected of having lived in a family before their arrival at the shelter (10 males, 2 females, estimated age 8 months -5 years), and the other 11 dogs were suspected of being feral with much less exposure to humans ( 2 males, 9 females, estimated age 8 months -3 years). All testing was conducted in a $9 \times 9 \mathrm{~m}$ room at the shelter that was relatively unfamiliar to the subjects.

All subjects were taken to the testing room and tested by two unfamiliar experimenters. Subjects were given time to explore the new room and become comfortable with the experimenters before testing began. All subjects were first given warm-up trials to introduce them to the 'hiding game'. Subjects watched as an experimenter hid food in one of two containers. The subjects were then released to find the food that they had seen hidden. A correct choice was scored when a dog touched the baited cup first, while an incorrect choice was scored when the dog touched the empty cup first. If a dog did not clearly touch one of the two cups within $25 \mathrm{~s}$ (i.e. the dog became distracted or unmotivated), the trial was repeated. Subjects were allowed to continue to the test session only after they selected the baited bowl in four consecutive trials (two for each side). All dogs met this criteria in no more than eight trials. Once the warm-ups were completed, each subject participated in the test. The test followed the procedure of the warm-up except that we used an opaque board $(55 \times 33 \mathrm{~cm})$ to occlude the baiting process from the onlooking subject, so that the subjects could see the food, but they could not see the cup in which the food was hidden.

The general procedure of the test involved one experimenter (E1) placing two hiding locations $1 \mathrm{~m}$ apart from each other and $1.5 \mathrm{~m}$ from a leashed subject held by E2. Once the subject was watching (E1 attracted her attention by showing her food), food was hidden in one of the two hiding locations. Once the baiting was completed, E1 gave one of four cues to indicate the location of the hidden food to the subject: (1) Point: E1 pointed repeatedly towards the correct cup four times with an extended index finger while alternating her gaze between the subject and the cup. The distance between index finger and cup was approximately $20 \mathrm{~cm}$; (2) Mark: E1 placed a black and white coloured piece of wood $(11 \times 7 \times 3 \mathrm{~cm})$ on the top of the cup with food. During the placement of the marker, E1 looked in the direction of the baited food cup; (3) Shake correct: E1 picked up the baited cup and shook it such that the food inside made an audible noise; (4) Shake empty: E1 picked up the empty cup and shook it (no noise was produced since it was empty).

Each subject received eight trials with each cue for a total 32 trials. The different cues were presented across the 32 trials in a randomized order with the stipulation that the same condition was never given in three consecutive trials. Subjects received four sessions of eight trials each, with at least $10 \mathrm{~min}$ between sessions. The location of the food was counterbalanced with the stipulation that food was never placed in the same hiding location for three consecutive trials. One subject passed the warm-ups but later chose not to participate (i.e. it did not make a choice by touching one of the cups during the test, even when tested again on a different day) and was completely dropped from the analysis. Less than $4 \%$ of trials had to be repeated because of the subject's lack of participation, giving a mean of 1.22 repeats per subject. A second coder scored $23 \%$ of the trials from video to assess interobserver reliability, which was excellent (Cohen's kappa $=0.99, N=160$ ).

\section{Results and Discussion}

Figure 4 presents the mean percentage of correct choices towards the cued cup for the four types of cues. Dogs used all cues at above-chance levels (one-sample $t$ tests: point: $t_{22}=7.3$, $P<0.001$; mark: $t_{22}=5.4, P<0.001$; shake correct: $t_{22}=9.1$, $P<0.001$; shake empty: $\left.t_{22}=-5.0, P<0.001\right)$. There was no significant difference in performance between family-reared or feral shelter dogs for any of the cues tested (independent sample $t$ test), and both groups used all cues at above-chance levels (one-sample $t$ tests: $P<0.05$ ).

These results demonstrate clearly that experimentally naïve shelter dogs, regardless of the suspected background, are skilled at using a variety of human cues, including novel communicative cues (e.g. a physical marker on a baited cup). Importantly, compared to chance, subjects were even more likely to approach an empty hiding location if a human had previously shaken it (regardless of whether it made noise or contained food). While this replicates the 


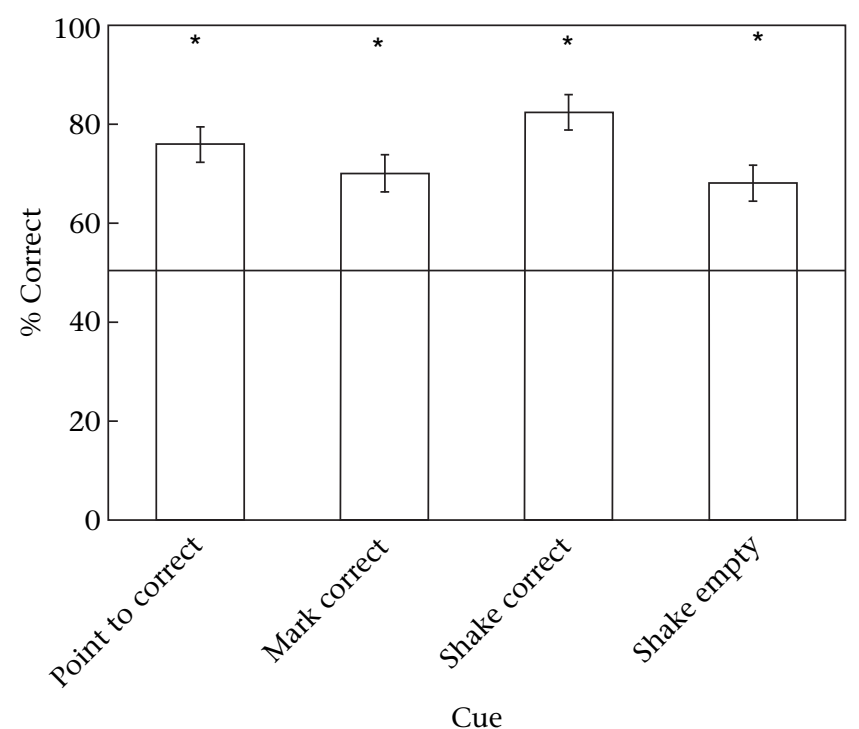

Figure 4. Mean \pm SE percentage of correct choices by 22 naïve shelter dogs for the cup that was cued by an unfamiliar human using one of four cues. The line represents the level of chance performance. ${ }^{*} P<0.05$ (one-sample $t$ tests comparing each group to chance or $50 \%$ ).

finding that dogs may not have a deep causal understanding of the physical properties of containers (Bräuer et al. 2006), it also shows that the dogs in our study understood that the human cues that we provided were communicative. Finally, these findings support the consensus in the literature that while dogs may vary in the degree to which they use human communicative cues, the vast majority are capable of using these cues to find hidden food at levels above chance.

\section{RESPONSE TO WYNNE ET AL. (2008): DOES HUMAN EXPERIENCE MATTER?}

Wynne et al. (2008) challenged studies showing that young dog puppies use basic communicative cues (i.e. pointing gestures) virtually as well as older dogs that obviously have more human experience. They focused their critique on Riedel et al. (2008), who reported in three experiments that puppies as young as 6 weeks old are skilled at using human communicative cues. First, Riedel et al. (2008) reported a longitudinal comparison of performance in dogs, aged 6-24 weeks old, with two human pointing cues, a novel marker cue, and a control condition with no cue. Using a factorial analysis, the authors reported that (1) even the youngest puppies were skilled at all of these communicative cues and (2) there was no evidence for a difference in performance between the different age groups with the various cues. Moreover, Riedel et al. (2008) conducted two additional studies demonstrating that dog puppies are not simply attracted to the hand of the experimenter but actually understand the communicative nature of the gesture across age groups.

Wynne et al. (2008) raised two concerns regarding this study. First, they questioned the conclusion that age does not affect dogs' comprehension of pointing in a major way, attributing the lack of an age effect to a lack of statistical power. Second, they argued that the choice of subjects was not well suited to find such an age effect in the first place and a simple learning mechanism explained the puppies' success at all ages. We discuss each issue in turn.

First, Wynne et al. (2008) reanalysed Riedel et al.'s (2008) data and, after removing the control condition from the factorial analyses, found a stronger age effect than was originally reported. Their justification for removing the control condition was that age would not have affected the control condition in the same way as the experimental conditions, and so its inclusion may have lowered the dogs' overall performance. Note, however, that in the original paper the experimental conditions were already analysed separately and it was found that only the marker condition, but not the two pointing conditions, changed with age (Riedel et al. 2008, page 1007). Therefore, the age effect reported by Wynne et al. may have been mostly due to the positive influence of the marker condition. Reanalysing the data with only the two pointing cues (after removing the marker and control conditions) again revealed no age effect $\left(F_{1,3}=2.23, P=0.094\right)$. Thus, if the control condition lowered the overall score, then the marker condition increased the pointing cues' score, something that our original analysis of each communicative cue separately had already established.

Moreover, experiments 2 and 3, which Wynne et al. (2008) did not discuss in their commentary, showed the same overall result: 6-week-old puppies did not differ from adult dogs in the comprehension of distal pointing cues. Pooling the results on following pointing cues from these last two experiments and comparing 6 -week-old puppies to adult dogs showed no age effect (independent sample $t$ test: $t_{78}=1.756, P=0.083$ ), and indeed the puppies were numerically higher (mean correct choices: 6 -week-olds: 5.06; adults: 4.54). As noted in our original paper, the only major age effect appeared in the marker condition of study 1, where the correct cup was marked with an object placed on top of it. One hypothesis is that this marker condition may test something other than dogs' response to communication. That is, dogs may be interested in exploring the marker itself, and this interest may increase with age, while their ability to comprehend communicative cues stays constant.

Second, Wynne et al. (2008) also suggested the possibility that stronger age effects might have been observed if puppies younger than 6 weeks old had been tested. They proposed testing puppies as young as 4 weeks old. While this is a theoretical possibility, we argue that it is extremely unlikely that a valid test can be conducted when working with puppies this young. Four-week-old puppies have poor motor control and eyesight (if their eyes are even open), putting them at a distinct disadvantage in the test for reasons unrelated to their ability to understand communicative gestures. An alternative might be to rear two groups of puppies with differential experience with humans. But when Hare et al. (2002) compared litter-reared puppies (with almost no human contact besides routine care) to family-reared puppies, they found no effect of rearing history.

Finally, Wynne et al. (2008) also suggested the possibility that subjects form a simple association between the hand and food that allows them to use the pointing gesture. However, in proposing such an explanation the authors have failed to acknowledge the findings of experiments 2 and 3 of Riedel et al. that were designed explicitly to test this hypothesis and rule it out as an explanation. In these two experiments, the degree to which the puppies could use the human's hand to find the food was substantially reduced because the hiding cups were placed next to the dog instead of next to the experimenter. Six-week-old puppies readily used this cue at levels well above chance and their performance was comparable to that of adult dogs. In addition, a separate analysis showed that puppies were not more successful if they had approached the human's hand first, indicating that they could not solve this problem merely by using the hand as a spatial cue.

In summary, there is currently little evidence that significant exposure to humans is necessary for dog puppies to reliably use human communicative cues. We doubt that younger puppies would provide an appropriate test, and controls have already ruled out the low-level explanation that the performance is a result of a simple attraction to human hands. 


\section{GENERAL DISCUSSION}

Based on our reanalysis and new experiment, we do not believe that Udell et al. (2008) or Wynne et al. (2008) provide compelling evidence that challenges the domestication hypothesis. First, and most importantly, our reanalysis of their data examining participation and correct choice measures separately provided no evidence that wolves outperform dogs in using human communicative cues. Moreover, our experiment examining the use of three different human communicative cues by a larger sample of shelter dogs, using a more conventional method, showed that even shelter dogs were skilled at using a variety of human communicative cues (including cues that are probably novel to them). Finally, in reanalysing our own data from Riedel et al. (2008), we again found no compelling evidence for age differences in the use of a human pointing cue that Wynne et al. (2008) argued exists. We also point out that experiments 2 and 3 from our study already ruled out the low-level alternative explanations suggested by Wynne et al. (2008).

While our reanalysis and new data highlight many of the central flaws of these two critiques, there are other reasons to doubt that the data presented in Udell et al. (2008) provide a strong test of the domestication hypothesis. Perhaps most importantly, Udell and colleagues did not rear the different groups of canids for the purpose of their experiments. Consequently, they were unable to rule out differential rearing histories as an explanation, unlike other studies that have used canids specifically reared for this type of experiment (wolves/dogs: Viranyi et al. 2008; foxes: Hare et al. 2005). In fact, we suspect that, given their use in public education programmes, the wolves that Udell and colleagues tested probably had received previous training and were highly socialized. This socialization probably gave their subjects significant experience responding to actions similar to human pointing, whether the animal handler was aware of this type of exposure or not. Udell et al. (2008) cannot rule out this type of simple exposure explanation for the success of their adult wolves based on the current data. In contrast, the wolves tested in Hare et al. (2002) had no formal training as adults with clickers, pointing gestures, or anything else, while the wolves tested by Viranyi et al. (2008) only skilfully used distal pointing cues after intense socialization and explicit training requiring dozens of trials. Overall, then, given that Hare et al. (2005) and Viranyi et al. (2008) reared the foxes, wolves and dogs to control for exposure to humans, we argue that these previous comparisons provide the most powerful data regarding the effect of domestication on canid social skills.

Another concern regarding Udell et al.'s (2008) study is that their subjects were only tested using a single type of pointing cue. Without testing wolves with novel gestures, it is unclear how flexibly they can use human gestures. In contrast, dogs flexibly use novel cues and even iconic cues (e.g. Kaminski et al., in press). This means that, while it is possible, even likely, that learning is important in the development of some of dogs' social skills for interacting with humans, when dogs are presented with novel communicative problems, they generalize their learned skills to new situations. Therefore, even if learning plays a role in the development of these skills, this does not mean that the domestication hypothesis is totally incorrect, or that richer cognitive explanations of dogs' skills are not warranted. Learning explanations are traditionally used as alternative explanations to flexible understanding or more cognitive interpretations of animal behaviour, but there is no a priori reason to suggest that learning and more high-level cognitive explanations are mutually exclusive (Call 2001). Therefore, in conclusion, we suggest that the findings we have available now remain completely consistent with the hypothesis that the unusual skills of domestic dogs in using human communicative cues most likely evolved during and as a result of human domestication.

We thank Kara Schroepfer for her comments, Katrin Schumann for help with data collection, Monique Udell and Clive Wynne for providing us with the raw data from their study, and Clive Wynne for providing comments on an earlier version of this manuscript. All experiments were conducted in accordance with the laws governing the ethical treatment of animals in Germany.

\section{References}

Agnetta, B., Hare, B. \& Tomasello, M. 2000. Cues to food location that domestic dogs (Canis familiaris) of different ages do and do not use. Animal Cognition, $\mathbf{3}$ 107-112.

Bräuer, J., Kaminski, J., Riedel, J., Call, J. \& Tomasello, M. 2006. Making inferences about the location of hidden food: social dog, causal ape. Journal of Comparative Psychology, 120, 38-47.

Call, J. 2001. Chimpanzee social cognition. Trends in Cognitive Sciences, 5, 388-393.

Cooper, J. J., Ashton, C., Bishop, S., West, R., Mills, D. S. \& Young, R. J. 2003. Clever hounds: social cognition in the domestic dog (Canis familiaris). Applied Animal Behaviour Science, 81, 229-244.

Hare, B. \& Tomasello, M. 2005. Human-like social skills in dogs? Trends in Cognitive Sciences, 9, 439-444.

Hare, B., Call, J. \& Tomasello, M. 1998. Communication of food location between human and dog (Canis familiaris. Evolution of Communication, 2, 137-159.

Hare, B., Brown, M., Williamson, C. \& Tomasello, M. 2002. The domestication of social cognition in dogs. Science, 298, 1634-1636.

Hare, B., Plyusnina, I., Ignacio, N., Schepina, O., Stepika, A., Wrangham, R. \& Trut, L. 2005. Social cognitive evolution in captive foxes is a correlated by-product of experimental domestication. Current Biology, 16, 226-230.

Kaminski, J., Tempelmann, S., Call, J. \& Tomasello, M. In press. Domestic dogs comprehend human communication with iconic signs. Developmental Science.

Miklosi, A. 2008. Dog Behavior, Evolution \& Cognition. New York: Oxford University Press.

Miklosi, A. \& Soproni, K. 2006. A comparative analysis of animals' understanding of the human pointing gesture. Animal Cognition, 9, 81-93.

Miklosi, A., Kubinyi, E., Topal, J., Viranyi, Z. \& Csanyi, V. 2003. A simple reason for a big difference: wolves do not look back at humans, but dogs do. Current Biology, 13, 763-766.

Riedel, J., Schumann, K., Kaminski, J., Call, J. \& Tomasello, M. 2008. The early ontogeny of human-dog communication. Animal Behaviour, 75, 1003-1014.

Soproni, K., Miklosi, A., Topal, J. \& Csanyi, V. 2001. Comprehension of human communicative signs in pet dogs (Canis familiaris). Journal of Comparative Psychology, 115, 122-126.

Udell, M. A. R., Dorey, N. R. \& Wynne, C. D. L. 2008. Wolves outperform dogs in following human social cues. Animal Behaviour, 76, 1767-1773.

Viranyi, Z., Gacsi, M., Kubinyi, E., Topal, J., Belenyi, B., Ujfalussy, D. \& Miklosi, A. 2008. Comprehension of human pointing gestures in young human-reared wolves (Canis lupus) and dogs (Canis familiaris). Animal Cognition, 11, 373-387.

Wynne, C. D. L., Udell, M. A. R. \& Lord, K. A. 2008. Ontogeny's impact on humandog communication. Animal Behaviour, 76, e1-e4. 